\title{
Novel regulation and functional interaction of polycistronic miRNAs
}

\author{
MARY TRUSCOTT, ${ }^{1}$ ABUL B.M.M.K. ISLAM, ${ }^{\mathbf{2}}$ and MAXIM V. FROLOV ${ }^{\mathbf{1}}$ \\ ${ }^{1}$ Department of Biochemistry and Molecular Genetics, University of Illinois at Chicago, Chicago, Illinois 60607, USA \\ ${ }^{2}$ Department of Genetic Engineering and Biotechnology, University of Dhaka, Dhaka 1000, Bangladesh
}

\begin{abstract}
The importance of microRNAs in gene expression and disease is well recognized. However, what is less appreciated is that almost half of miRNA genes are organized in polycistronic clusters and are therefore coexpressed. The mir-11 998 cluster consists of two miRNAs, miR-11 and miR-998. Here, we describe a novel layer of regulation that links the processing and expression of miR-998 to the presence of the mir-11 gene. We show that the presence of miR-11 in the pri-miRNA is required for processing by Drosha, and deletion of mir-11 prevents the expression of miR-998. Replacing mir-11 with an unrelated miRNA rescued miR-998 expression in vivo and in vitro, as did expressing miR-998 from a shorter, more canonical miRNA scaffold. The embedded regulation of miR-998 is functionally important because unchecked miR-998 expression in the absence of miR-11 resulted in pleiotropic developmental defects. This novel regulation of expression of miRNAs within a cluster is not limited to the mir-11 998 cluster and, thus, likely reflects the more general cis-regulation of expression of individual miRNAs. Collectively, our results uncover a novel layer of regulation within miRNA clusters that tempers the functions of the individual miRNAs. Unlinking their expression has the potential to change the expression of multiple miRNA targets and shift a biological response.
\end{abstract}

Keywords: polycistronic miRNA; miRNA cluster; cis-regulation of miRNA expression; miRNA biogenesis

\section{INTRODUCTION}

MicroRNAs (miRNAs) are small noncoding RNAs that repress the expression of target protein-coding transcripts. miRNAs are initially expressed as hairpins embedded within a primary transcript. The pri-miRNA structure is recognized by the Drosha/Pasha microprocessor and cleaved to release a pre-miRNA stem-loop, which is exported from the nucleus for further processing into a mature, functional miRNA (Kim et al. 2009). miRNAs regulate a variety of biological processes including development, proliferation, cell death, metabolism, and tissue homeostasis. Although earlier studies identified the stronger and more recognizable phenotypes of a few miRNAs (e.g., let-7), the majority of miRNAs exert subtle effects. It has been suggested that this is because miRNAs function to dampen noise arising from the stochastic nature of gene expression or raise the threshold required to elicit a response (Hornstein and Shomron 2006; Ebert and Sharp 2012; Ameres and Zamore 2013; Schmiedel et al. 2015). This may explain why it has been so difficult to assign a clear-cut function to many miRNAs and why inactivation of a particular miRNA rarely gives rise to severe defects.

miRNAs can be expressed from a separate transcript, they can be expressed from a transcript encoding both a miRNA

Corresponding author: mfrolov@uic.edu

Article published online ahead of print. Article and publication date are at http://www.rnajournal.org/cgi/doi/10.1261/rna.053264.115. and a protein-coding gene, and they can be generated from a primary transcript encoding more than one miRNA (polycistronic). This latter case of miRNAs being expressed from clusters accounts for $\sim 40 \%$ of miRNAs (Kim et al. 2009). The reason for and implications of such a genomic organization are not thoroughly understood. However, polycistronic clusters were shown to give rise to mature miRNAs that regulate overlapping or complementary sets of targets to elicit a biological response. There is mounting evidence that although the miRNAs from a cluster are processed from a common polycistronic RNA, their biogenesis can be differentially regulated and give rise to changes in relative levels of the mature miRNAs. The mechanism behind such regulation and settings where it is important are active areas of research.

The mir-11 998 cluster consists of two miRNAs, miR-11 and miR-998, and it is embedded within the Drosophila E2f1 gene. Both miRNAs are coexpressed with the host gene E2f1 and were shown to modulate the apoptotic function of E2f1, albeit by different mechanisms. Here we report a novel layer of regulation between miR-11 and miR-998. Unexpectedly, the processing and expression of miR-998 strongly depends

\footnotetext{
( 2015 Truscott et al. This article is distributed exclusively by the RNA Society for the first 12 months after the full-issue publication date (see http://rnajournal.cshlp.org/site/misc/terms.xhtml). After 12 months, it is available under a Creative Commons License (Attribution-NonCommercial 4.0 International), as described at http://creativecommons.org/licenses/by$\mathrm{nc} / 4.0 /$.
} 
on the presence of the mir-11 gene. As a result, pre- and mature miR-998 are undetectable in the absence of mir-11. This dependence of miR-998 expression on mir-11 is functionally important because breaking up this dependence and expressing miR-998 from its endogenous location in the absence of mir-11 resulted in pleiotropic developmental defects at high frequency. Importantly, this interdependence in the expression of miRNAs within a cluster is not limited to the mir11 998 cluster and likely reflects the more general cisregulation of expression of individual miRNAs.

\section{RESULTS}

\section{The expression of miR-998 is dependent on mir-11}

To begin to characterize the expression of individual miRNAs from the mir11 998 cluster we examined the levels of miR998 and miR-11 in their respective and reciprocal mutant alleles, the mir-11 deletion, mir-11 1 , and mir-998 exc222, a mir998 mutant allele generated by imprecise $P$-element excision (Truscott et al. 2011, 2014). As expected, in both quantitative RT-PCR (Fig. 1A) and Northern blot analysis (Fig. 1B),

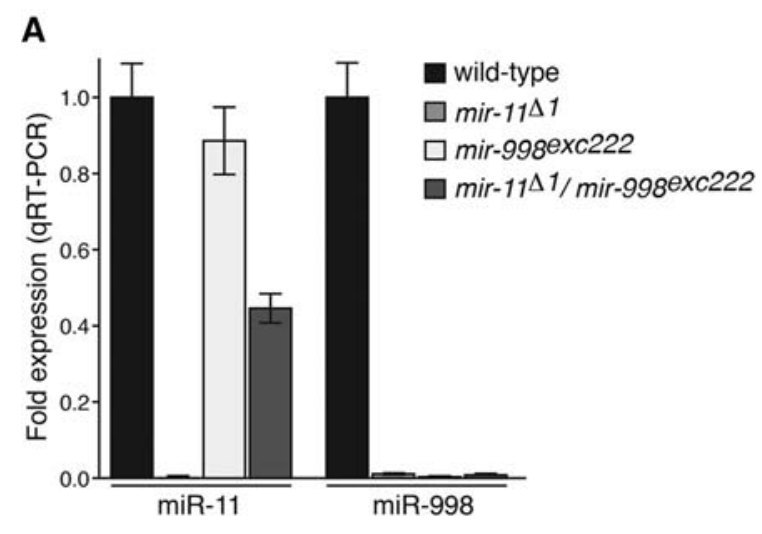

B

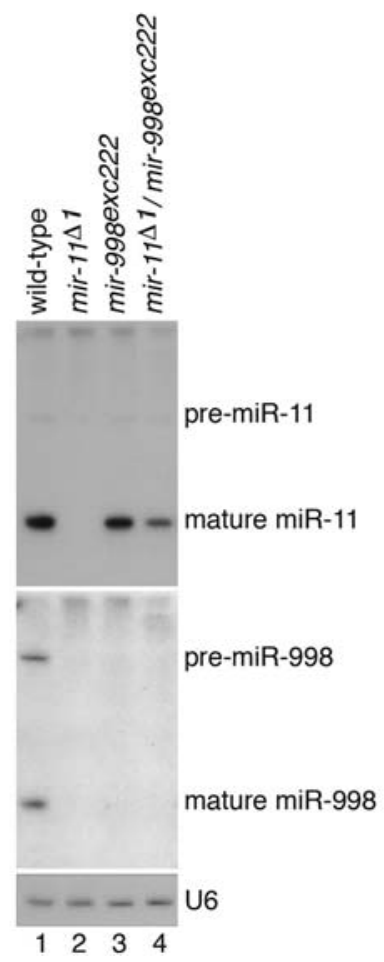

C
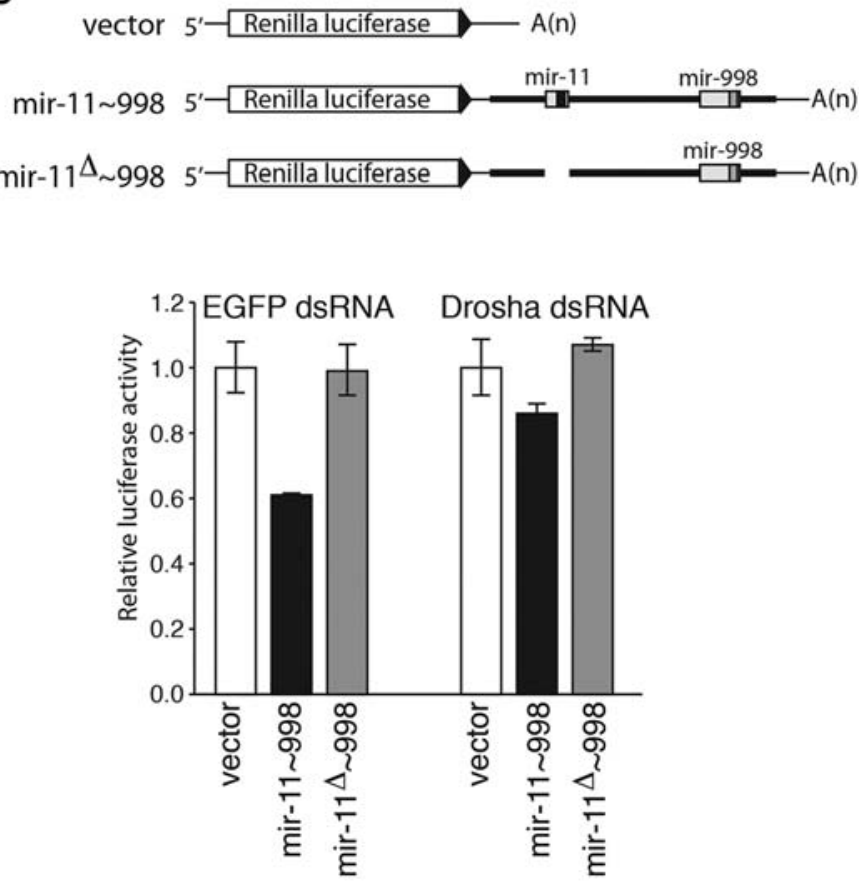

FIGURE 1. miR-998 expression is dependent on mir-11 in vivo. (A) Quantitative RT-PCR was performed on RNA from third instar larvae of the

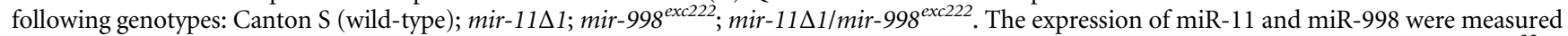
by TaqMan assay and normalized to miR-14. (B) RNA from the samples in $A$ was used in Northern blot analysis. Membranes were probed with ${ }^{32} \mathrm{P}-$ labeled miR-11, miR-998, or U6 anti-sense probes. $(C)$ S2R+ cells were soaked with EGFP dsRNA or Drosha dsRNA for 4 d, and then transfected with the following Renilla luciferase sensor plasmids: pGL2-hsp70 min-hRluc; pGL2-hsp70 min-hRluc-mir-11 998; pGL2-hsp70 min-hRluc-mir-11 $\Delta$ $\sim 998$. Cells were harvested $30 \mathrm{~h}$ after transfection, and Renilla luciferase activity was measured. Results are presented as the mean of three separate luciferase measurements from each of three biological replicates. Error bars represent standard error. 
miR-11 was expressed in wild-type animals, and in the mir$998^{\text {exc222 }}$ mutant, but not in the mir-11 deletion, mir-11 1 . Furthermore, miR-11 expression was reduced in the mir$11 \Delta 1 /$ mir-998 $8^{\text {exc222 }}$ trans-heterozygous combination, demonstrating the sensitivity of the measurements.

Surprisingly, miR-998 expression was not detected in mir$11 \Delta 1$ animals by qRT-PCR, even though the sequence of the mir-998 gene was intact (Fig. 1A). This result was confirmed by Northern blot analysis that showed the absence of the mature miR-998 in mir-11 1 animals (Fig. 1B). Significantly, no precursor miRNA of miR-998 (pre-miR-998) was detected, suggesting that the primary miRNA (pri-miR-998) is not processed in the absence of the mir-11 gene. Importantly, the expression of miR-11 in trans in mir-11 $1 /$ mir-998 $8^{\text {exc222 }}$ trans-heterozygous animals did not rescue miR-998 expression. Therefore, mir-11 is necessary in a cis configuration for the expression of miR-998, and the regulation occurs at the level of the pri-miRNA transcript.

\section{A pri-miRNA lacking the mir-11 gene is not processed by Drosha}

Results described above indicate that pri-miR-998 is not processed efficiently in the absence of the adjacent miR-11. Pri-miRNA transcripts are endonucleolytically processed by the Drosha/Pasha microprocessor complex, which binds the pri-miRNA in a manner that is dependent on secondary structure and possibly also on sequence (Lee et al. 2002, 2003; Han et al. 2006). To investigate whether the microprocessor processes miR-998 more efficiently in the presence of miR11 , we inserted the primary miR-11 998 transcript downstream from the luciferase gene in the $3^{\prime}$ UTR, and transfected these luciferase sensors in $\mathrm{S} 2 \mathrm{R}+$ cells. If the primary miRNA is processed by the Drosha/Pasha microprocessor, the luciferase transcript is destabilized and degraded, which leads to a decrease in luciferase activity. However, if the primary miRNA is not processed by the microprocessor, the luciferase activity would be comparable to a sensor without a pri-miRNA in its $3^{\prime}$ UTR (Fig. 1C).

The luciferase sensor containing the wild-type pri-miR11 998 in the $3^{\prime}$ UTR yielded less luciferase activity than the parental sensor control, which had no pri-miRNA in the $3^{\prime}$ UTR (Fig. 1C) indicating that the luciferase assay accurately detects processing of wild-type pri-miR-11 998. In contrast, a luciferase sensor containing pri-mir-11 $11 \sim 998$ sequence, which contains only the mir-998 gene, was expressed at the same level as the control transcript with no pri-miRNA in the $3^{\prime}$ UTR. Therefore, the mir-998 gene on its own had no effect on luciferase transcript stability (Fig. 1C). Inactivating the microprocessor complex by treating the cells with dsRNA against Drosha increased the luciferase activity of the sensor carrying the wild-type mir-11 998 primary miRNA, which is indicative of reduced processing of the miRNAs present in the $3^{\prime}$ UTR. However, the knockdown of Drosha had no effect on the sensor carrying only the mir-
998 gene without the adjacent mir-11. This suggests that miR998 is not processed by Drosha unless miR-11 is present. Together with the qRT-PCR and Northern blot analysis these results point to an unusual dependence of the processing of miR-998 on the presence of adjacent miR-11. When mir-11 was deleted, neither pre- nor mature miR-998 was expressed.

\section{miR-998 expression can be rescued by replacing mir-11 with a heterologous miRNA}

To gain further insight into the mechanism underlying the dependence of miR-998 on miR-11, we generated a series of constructs, expressed them using the baculovirus promoter in S2R+ cells, and followed the expression of mature miR998 by these constructs using qRT-PCR. In a control experiment, miR-998 was expressed when a wild-type, 1042-bp fragment containing miR-11 and miR-998 was transfected. Similarly to the in vivo effects, and in the $3^{\prime}$ UTR luciferase sensors (Fig. 1), miR-998 is not expressed when the miR11 stem-loop is deleted (Fig. 2A). Therefore, the insect cell culture system accurately recapitulates the effects observed in mutant animals, and confirms that a portion of the intron in which mir-11 998 is embedded contains the cis-regulatory sequence that modulates miR-998 expression.

The requirement of mir-11 for miR-998 expression may be context-, sequence-, or structure-specific. We first asked whether miR-11 was required upstream of mir-998 for miR998 expression. mir-11 and mir-998 sequences were swapped and miRNA expression was measured. As shown in Figure 2B, miR-998 was expressed regardless whether the mir-11 gene was upstream of mir-998 (mir-11 998) or downstream from mir-998 (mir-998 11). However, deletion of the mir-

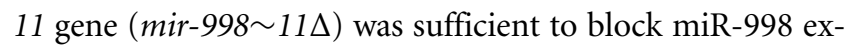
pression. Therefore, the relative order of the mir-11 and mir-998 genes does not influence the requirement of mir-11 for miR-998 expression. However, an intact mir-11 gene is required for miR-998 expression.

To determine whether the dependence of miR-998 expression is specific to the sequences in the mir-11 gene, mir-11 was replaced with a chimeric short hairpin miRNA (shmiR), mir-1/mCherry. miR-1/mCherry represses mCherry expression and was previously shown to be otherwise biologically inert (Haley et al. 2008). Strikingly, the presence of this heterologous miRNA was sufficient to rescue the expression of miR-998. Furthermore, similarly to the mir-11 deletion, truncating the mCherry shmiR hairpin also resulted in the loss of expression of miR-998 (Fig. 2A). Therefore, a miRNA is required nearby for the expression of miR-998, but the precise sequence of the miRNA is not important.

One reason that, on its own, mir-998 is not processed could be that its pre-miRNA scaffold is longer than the average (142 vs. $94 \mathrm{nt}$, respectively), which may serve as a poor substrate for the Drosha/Pasha microprocessor. To address this possibility, the mature miR-998 was expressed from a chimeric mir-1/ 998 shmiR scaffold. In this shorter, more canonical miRNA 

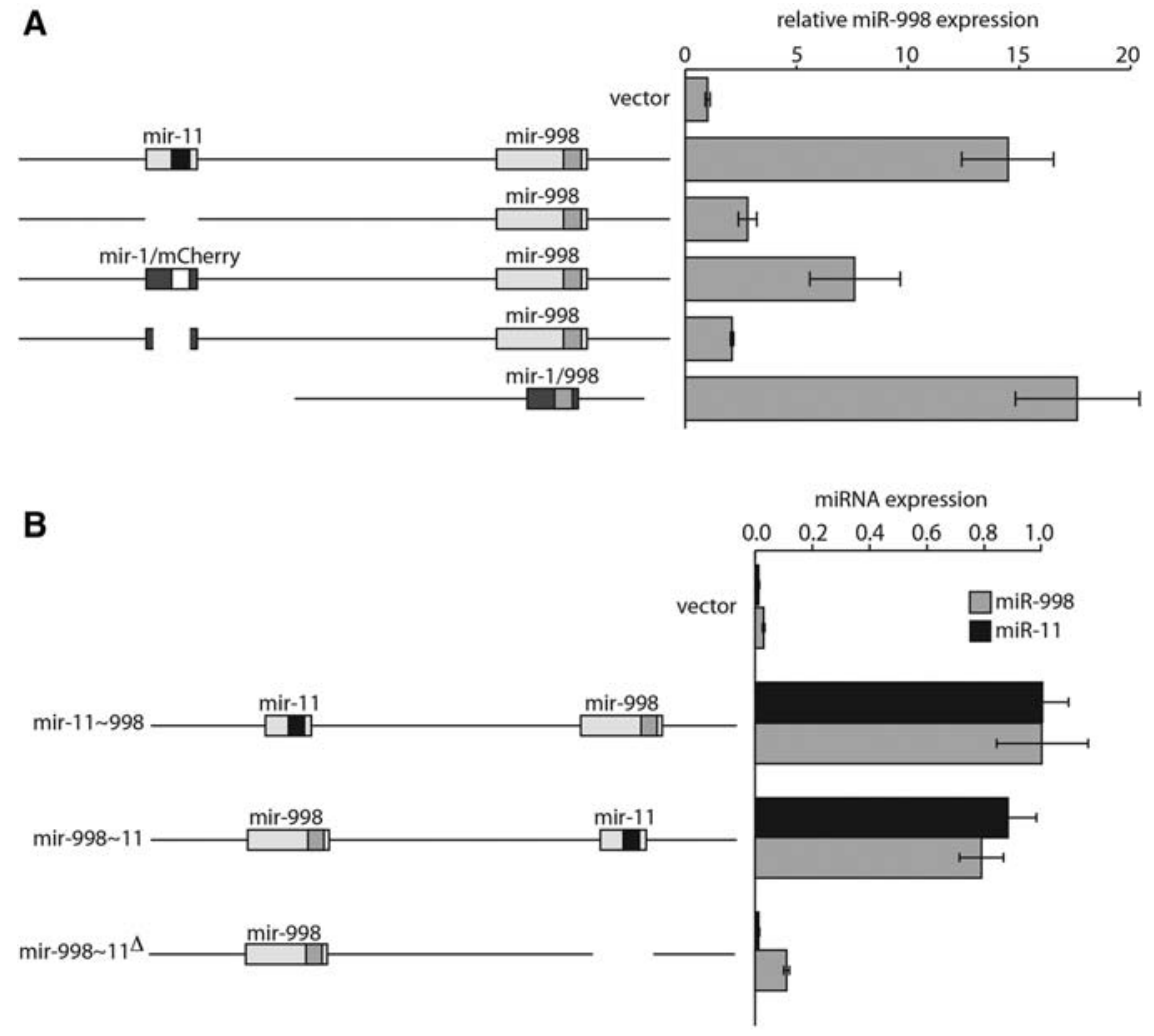

FIGURE 2. miR-998 expression is dependent on another miRNA nearby unless expressed from a shorter miRNA scaffold. S2R+ cells were transfected with constructs expressing wild-type and mutant $d E 2 f 1$ mir-11 998-containing intron sequences as shown in the diagram. RNA extracts were made $2 \mathrm{~d}$ after transfection and mature miR-998 $(A, B)$ and miR-11 $(B)$ were measured by TaqMan assay and normalized to miR-14 or bantam expression. Expression was normalized to empty vector control $(A)$ or wild-type mir-11 998 (B). Error bars represent standard error. serted into the mir-11 locus (Ge et al. 2012). Recombinase-mediated cassette exchange (RMCE) was used to replace the attP-flanked mini-white cassette in the mir-11 locus (Ge et al. 2012) with either a cassette containing mir-1/998 (mir-11 998 ${ }^{R M C E-1}$ ) or a cassette containing the $m$ Cherry shmiR and endogenous mir-998 (mir-11 998 ${ }^{\text {RMCE-2 }}$ ) (Fig. 3A). To confirm that miR-998 is expressed in these alleles, RNA was isolated from homozygous mutants and the level of miR-998 expression was determined by qRT-PCR. Although no miR-998 expression was detected in animals homozygous for the mir-11 deletion $\left(\operatorname{mir}-11^{K O(w-)}\right)$, in mir-11 998 $8^{R M C E-1}$ animals, miR-998 expression was 60\% that of wild-type animals, and in mir$11 \sim 998^{R M C E-2}$ allele homozygotes, miR998 was expressed at $30 \%$ of wild-type levels (Fig. 3B). Therefore, in agreement with the results in tissue culture cells, in vivo, miR-998 expression can be rescued by replacing the mir-11 gene with an exogenous shmiR or by expressing miR998 from the mir-1 scaffold. Importantly, these alleles express miR-998 at a level similar to the level of the endogenous miR-998. scaffold, miR-998 was expressed on its own without the need for a miRNA gene nearby (Fig. 2A). Therefore, the miR-998 gene requires an adjacent miRNA gene in order to be expressed, and this is due, at least in part, to its unusually large backbone, because replacing it with a chimeric miR-1 scaffold was sufficient to rescue miR-998 expression.

\section{Generation of $\operatorname{mir-11} \sim 998^{\text {RMCE }}$ knock-in alleles that express miR-998 in the absence of miR-11}

The striking dependence of miR-998 on miR-11 for expression raises the question of the functional significance of such an arrangement within the mir-11 998 cluster. To address this question, a series of targeted alleles in the mir$11 \sim 998$ cluster was generated that express miR-998 in its endogenous location but do not express miR-11. We took advantage of the results obtained in tissue culture cells (Fig. 2) that showed that replacing mir-11 with a heterologous miRNA ( $m$ Cherry shmiR) or substituting the long scaffold of miR-998 (mir-1/998) allows the expression of miR-998 in the absence of mir-11.

These alleles were targeted to a chromosome that contains an attP-flanked mini-white cassette that had been in-

\section{mir-11 ${ }^{998^{R M C E}}$ alleles suppress E2F-dependent cell death in $r b f$ mutant animals}

The mir-11 998 ${ }^{R M C E}$ alleles express miR-998 but do not express miR-11. Therefore, we began by characterizing these alleles in settings that are known to be sensitive to miR-998 expression but insensitive to miR-11 expression. One such context is the apoptotic response of $r b f$ mutant cells during eye development. In $r b f$ mutant eye discs, E2F activity is derepressed, which leads to EGFR-dependent ectopic cell death in a narrow stripe anterior to the morphogenetic furrow (MF) of larval eye discs (Fig. 3C; Moon et al. 2006). Overexpression of miR-998 was previously shown to suppress apoptosis, whereas the loss of mir-998 enhanced apoptosis in the MF of $r b f$ mutant larval eye discs. In contrast, overexpression of miR-11 had no effect on apoptosis (Truscott et al. 2014).

Clones of mir-11 998RMCE-1 homozygous mutant cells were induced in the $r b f$ mutant background and apoptotic cells were visualized with an antibody against active caspase-3. Notably, the high level of apoptosis in the morphogenetic furrow was strongly suppressed in mir-11 998 $8^{R M C-1}$ mutants (GFP negative) in comparison to the adjacent normal tissue (GFP positive). A similar result was obtained 
with other allele mir-11 998 ${ }^{R M C E-2}$ (Fig. 3C). The absence of cell death in the mir-11 998 ${ }^{R M C E}$ alleles was not the consequence of changes in the expression of Rbf or E2f1 (data not shown, and Truscott et al. 2011, 2014).

Both mir-11 998 ${ }^{R M C E}$ alleles express miR-998 in the absence of mir-11; therefore, the suppression of apoptosis could be due to the lack of mir-11 or due to the presence of miR998. To distinguish between these two possibilities, clones of mir- $11^{K O(w-)}$ mutant cells, which lack expression of both miR-11 and miR-998, were induced in rbf mutants, and apoptosis was examined by immunofluorescence. In contrast to strong suppression of apoptosis by the mir-11 998 ${ }^{R M C E}$
A

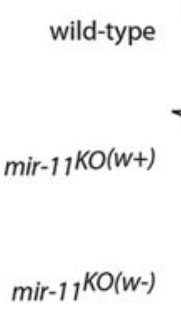

$d E 2 f 1$
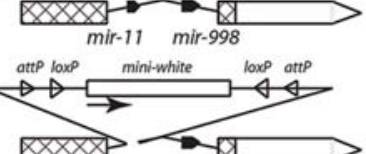

mir-998

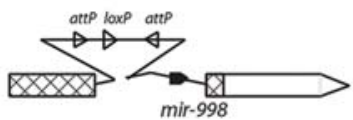

mir-11 998 $R M C E-1$

mir-11 998 RMCE-2
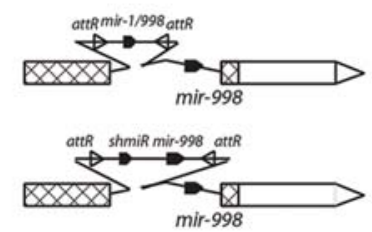

B

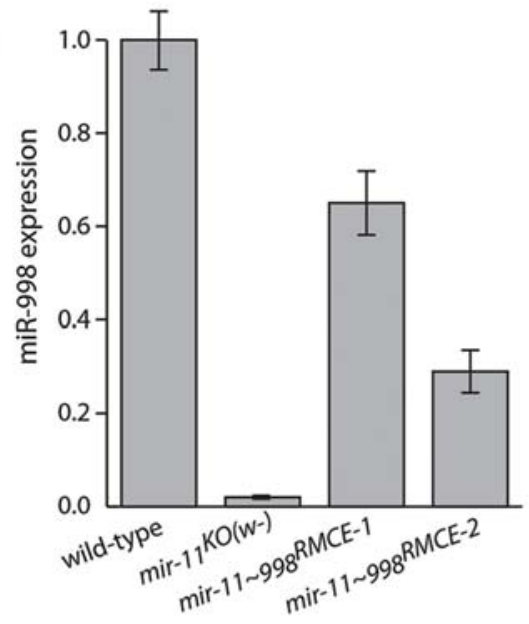

C

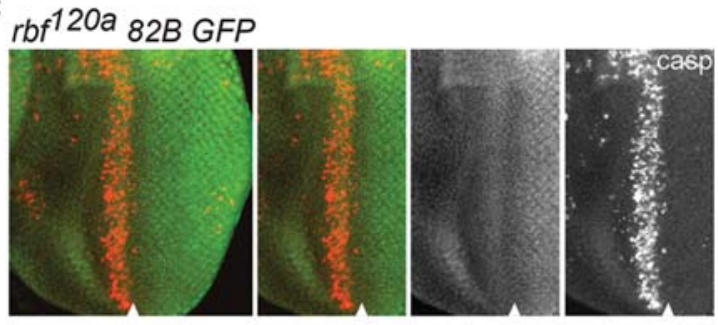

$r b f^{120 a} 82 B$ mir-11 998RMCE-1

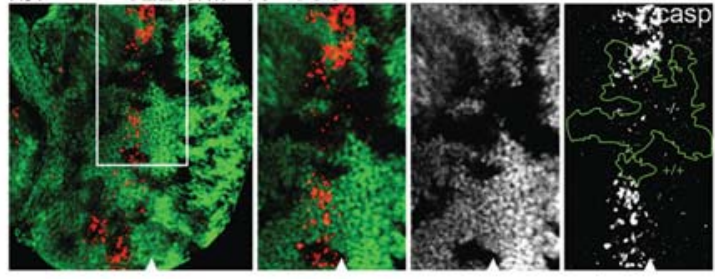

$r b f^{120 a} 82 B$ mir-11 998RMCE-2
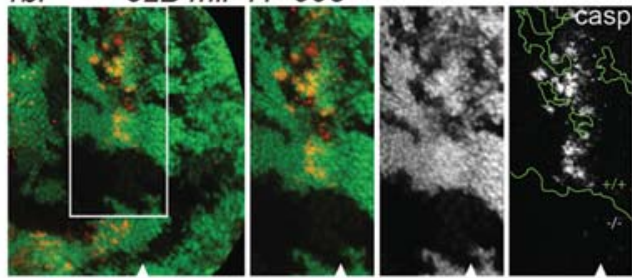

$r b f^{120 a} 82 B$ mir-11 $K O(w-)$

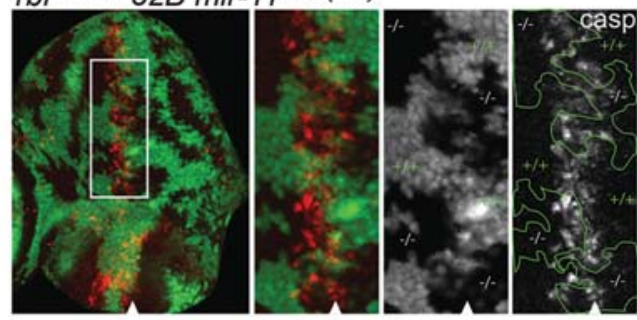

D

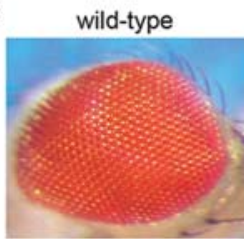

Elp, mir-11 998 RMCE-2

Elp, $\operatorname{mir}-11 \mathrm{KO}(\mathrm{w}-)$
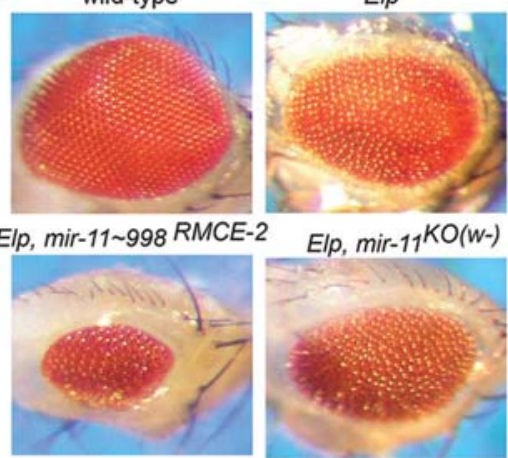

FIGURE 3. (Legend on next page) 
alleles that express miR-998 in the absence of miR-11, the combined loss of miR-998 and miR-11 had no effect on the number of apoptotic cells in the MF of $r b f$ mutant eye discs (Fig. 3C). These results strongly argue that the suppression of apoptosis by the mir-11 998 ${ }^{R M C E}$ alleles is due to the expression of miR-998. Thus, altering the normal balance between miR-11 and miR-998 in the mir-11 998 ${ }^{R M C E}$ alleles revealed that, in the absence of miR-11, unchecked miR998 provides a strong prosurvival signal to suppress apoptosis in $r b f$ mutants.

Given that miR-998 was shown to limit cell death in $r b f$ mutants by increasing EGFR signaling, we performed genetic interaction tests between the mir-11 998 ${ }^{R M C E}$ and EGFR alleles. The Ellipse allele is a gain-of-function allele of EGFR that results in a decrease in the number of ommatidial clusters and therefore a smaller eye. Consistently, the mir$11 \sim 998^{R M C E}$ alleles strongly enhanced the Ellipse phenotype, whereas mir- $11^{K O(w-)}$, which expresses neither miR-11 nor miR-998, had no effect (Fig. 3D).

Thus, we concluded that in the absence of miR-11, unchecked expression of miR-998 enhances EGFR signaling and protects $r b f$ mutant cells from apoptosis. These results are in agreement with the previous analysis of miR-998 transgenic animals, thus providing genetic evidence that the mir$11 \sim 998^{R M C E}$ alleles accurately reflect the function of miR-998.

\section{Expression of miR-998 in the absence of miR-11 results in pleiotropic effects and influences multiple phenotypic traits}

The results described above suggest that the unchecked expression of miR-998 in the absence of miR-11 elicited the effects in $r b f$ mutant cells that were not seen when miR-998 was coexpressed with miR-11. To determine the impact of unchecked miR-998 in normal animals, larval eye discs were dissected from a large cohort of the mir-11 998 ${ }^{R M C E}$ larvae and stained with ELAV antibodies to visualize differentiating photoreceptors. Surprisingly, we noted a wide range of abnormalities in mir-11 998 $8^{R M C E}$ animals, yet almost no strong defects were observed in animals mutant for mir998 only, or both mir-11 and mir-998. The phenotypes associated with the mir-11 998 ${ }^{R M C E}$ alleles included moderate and significant tissue remodeling (e.g., ectopic morphogenet- ic furrow formation, tubular projections), whereas milder defects included changes in composition, spacing, or orientation of ommatidia and delayed morphogenetic furrow movement (summarized in Fig. 4A, with examples in Fig. 4B). Thus, expression of miR-998 in the absence of miR-11 exerts pleiotropic effects that appear to affect various phenotypic traits. Consistent with this interpretation, overexpression of miR-998 using the Gal4/UAS system gave rise to various developmental defects. Expression of miR-998 in the dorsal half of the larval eye disc ( $D E>$ mir-998) delayed the MF progression and led to projections resembling antennal discs. Similarly, $p t c>$ mir-998 eye discs led to what appear to be ectopic antennae in $>50 \%$ of slow-developing animals (Fig. 4C).

To further characterize the mir-11 998 ${ }^{R M C E}$ mutant animals, we performed RNA sequencing and compared changes in gene expression in the eye discs of the mir-11 998 ${ }^{R M C E}$ alleles and the mir- $11^{K O(w-)}$ allele, which expresses neither miR-11 nor miR-998. Notably, when compared with wildtype, there was a higher number of differentially expressed (DE) genes in the mir-11 998 ${ }^{R M C E}$ mutants than in the mir- $11^{K O(w-)}$ mutants, and more GOBP categories were enriched in the mir-11 998 ${ }^{R M C E}$ versus WT samples compared with mir-11 ${ }^{K O\left(w^{-}\right)}$versus WT (Fig. 4D; Supplemental Table S1). GOBP enrichment analysis using the list of DE genes in the mir-11 998 ${ }^{R M C E}$ alleles that are also predicted miR-998 targets revealed overrepresentation of GOBP categories such as cell fate commitment and polarity. Importantly, such representation was not observed in mir-998 mutants nor in mutants lacking both miR-11 and miR-998 (Fig. 4D; Truscott et al. 2014). Thus, in agreement with the pleiotropic nature of phenotypic traits associated with mir-11 $998^{R M C E}$ alleles, genome-wide expression analysis revealed enrichment for various GOBP categories that may be associated with such phenotypes.

\section{The interdependence of expression in miRNA clusters is not limited to mir-11 998}

The results described above raise the question whether this type of regulation is specific to mir-11 998 or whether there are other clusters of coexpressed miRNAs that demonstrate interdependence of miRNA expression. To test this idea, we selected the highly evolutionarily conserved the mir-

FIGURE 3. mir-11 998 ${ }^{R M C E}$ alleles suppresses E2F-dependent cell death in $r b f$ mutant animals. (A) Diagrams of the different mutant alleles in the context of the $d E 2 f 1$ locus. mir- $11^{K O(w+)}$ contains an RMCE-competent cassette, was targeted to, and replaced the mir-11 gene (see Ge et al. 2012 for more details). mir-11 ${ }^{K O(w-)}$ is a mir-11 deletion generated by Cre recombinase-mediated deletion of sequences between loxP sites (Ge et al. 2012). mir-11 998 ${ }^{R M C E-1}$ and mir-11 998 ${ }^{R M C E-2}$ are the products of RMCE-mediated replacement of the mini-white cassette with E2f1 intron sequences in which the mir-11 gene was replaced with mir-1/998 or the mir-1/mCherry shmiR with wild-type mir-998 described in Figure 2. (B) miR-998 expression was measured in adult heads of flies with the indicated genotypes by qRT-PCR and TaqMan assay. miR-998 expression was normalized to $r$ 49. Results are expressed relative to wild-type control. Error bars represent standard error. $(C)$ Clones of indicated mutant tissue were generated using ey-FLP in $r b f^{120 a}$ hemizygous animals. The full genotypes are $r b f^{120 a} e y-F L P / Y$; FRT 82B Ubi-GFP/FRT 82B Ubi-GFP, rbf $f^{120 a} e y$ FLP/Y; FRT 82B mir-11 998 ${ }^{R M C E-1} / F R T$ 82B Ubi-GFP, rbf ${ }^{120 a}$ ey-FLP/Y; FRT 82B mir-11 998 ${ }^{R M C E-2} / F R T$ 82B Ubi-GFP, and rbf ${ }^{120 a}$ ey-FLP/Y;

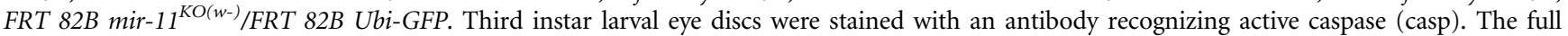
disc is shown in the left panel, and a higher magnification is shown in panels on the right. A minimum of 10 larvae were analyzed for each genotype, and representative results are shown. (D) Images of adult eyes from wild-type control, Egfr ${ }^{E l p} /+, E g f r^{E l p} /+; F R T 82 B, m_{i r-11 \sim 998^{R M C E-2}}$, and Egfr ${ }^{E l p} /+$; FRT $82 B$, mir- $11^{K O(w-)}$ animals. Images were taken at the same magnification. 
A

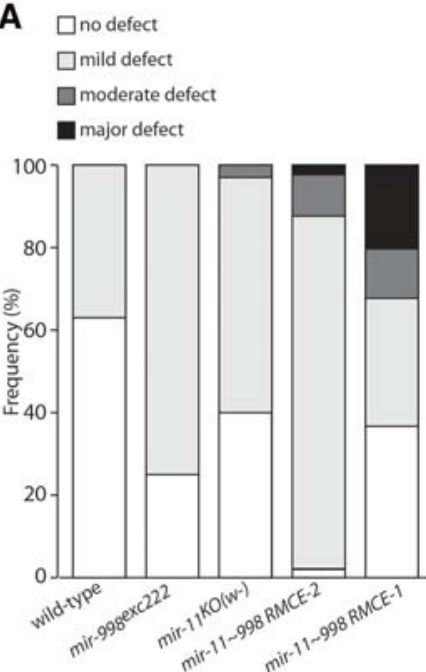

B
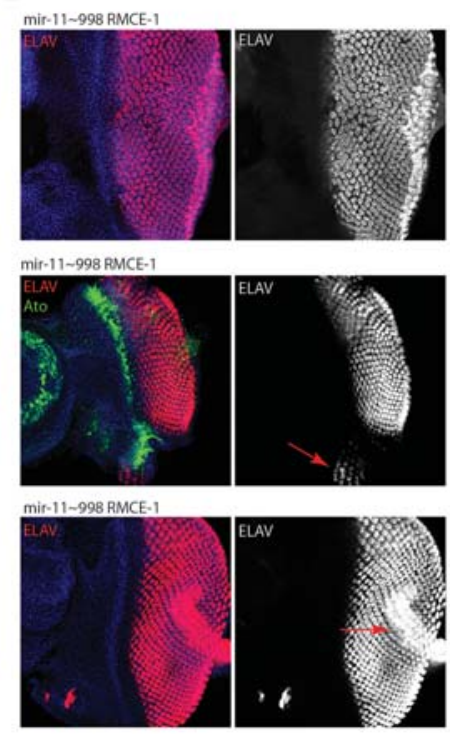

ey-Flp; 828, mir-11 998 RMCE.
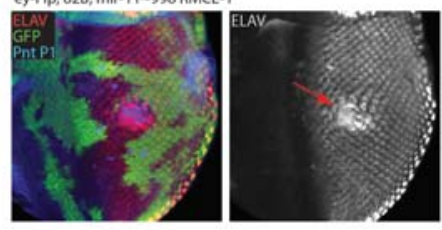

C
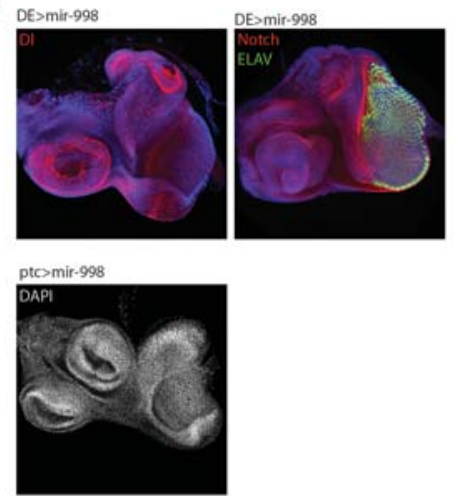

D
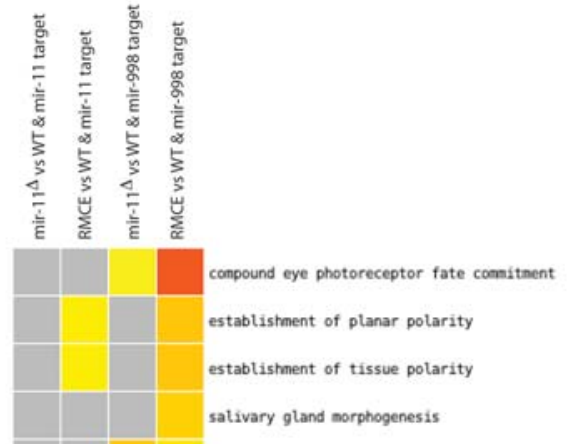

actin cytoskeleton organization

actin filament-based process

actin polymerization or depolymerization

induction of programed cell death by ecdysone

head segnentation

response to ecdysone

induction of programed cell death by hornones

R7 cell fate comitnent

interceltular bridge organization

inaginal disc fusion, thorax closure

R3/RA cell fate connitnent

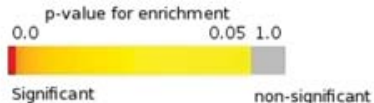

FIGURE 4. Unchecked miR-998 activity leads to pleiotropic defects. (A) Third instar larval eye discs of the genotypes indicated were dissected and stained with anti-ELAV, anti-Ato, or anti-Pnt P1 antibodies. Changes in developmental patterning were assessed and scored as having either no, mild, moderate, or major defect. (B) Examples of defects observed and summarized in $A$. $(C)$ Expression of miR-998 using the Gal4-UAS system. Third instar larval eye discs were dissected and stained with the antibodies indicated. A minimum of 10 larvae per genotype were assessed, a penetrance of phenotype $>50 \%$. $(D)$ Heat map of GOBP enrichment analysis of genes differentially expressed in the indicated genotypes that are also predicted miR-998 targets. Categories associated with pleiotropic defects are shown. Full GOBP enrichment analysis is listed in Supplemental Table S1.

100 let-7 125 miRNA cluster. Notably, like the configuration of mir-11 998, mir-125, the $3^{\prime}$-most miRNA in the mir-100 let-7 125 miRNA cluster, is longer than the aver- age miRNA (109 vs. $94 \mathrm{nt}$, respectively). We therefore asked whether the expression of miR-125 is dependent on the other miRNAs in the cluster. The miR$100 \sim$ let-7 $\sim 125$ cluster was expressed in $\mathrm{S} 2 \mathrm{R}+$ cells, along with deletions of either mir-100 or let-7 or of mir-100, let-7, and the intervening sequence (Fig. 5). The expression of miR-125 did not vary significantly between the intact cluster and the two single miRNA deletions. This was in agreement with previous work investigating expression in vivo in genomic rescue constructs in which individual miRNAs were deleted (Sokol et al. 2008). However, deletion of both mir100 and let-7 resulted in a threefold decrease in miR-125 expression. Therefore, like miR-998, miR-125 expression is enhanced by the presence of additional miRNA genes upstream, although the effect is not as dramatic as the one observed in the mir-11 998 cluster. Nevertheless, these results suggest that interdependence between miRNAs in clusters extends beyond the mir11 998 cluster.

\section{DISCUSSION}

Here, we describe a novel layer of posttranscriptional regulation of polycistronic miRNAs, as illustrated by the mir11 998 cluster. Most clustered miRNAs are processed from a single polycistronic RNA and, therefore, they are often coexpressed. Unexpectedly, the processing of miR-998 is strongly dependent on the presence of mir-11. The cis-regulatory role of miR-11 ensures that pre-miR-998 is only expressed if pre-miR-11 is expressed. Therefore, miR-998 expression is kept in check by miR-11.

Although mir-11 is required for the expression of miR-998, the converse is not true as mir-998 is not required for miR11 expression. The asymmetry in this intrinsic regulation may be explained in part by the longer than average length of the miR-998 pre-miRNA, 142 nt. Although Drosophila miRNAs range in length from 53 to $214 \mathrm{nt}$, almost $60 \%$ of miRNAs are within $10 \mathrm{nt}$ of the average length of 94 (Kozomara and Griffiths-Jones 2011). pri-miRNA cleavage by the microprocessor complex is determined by and 

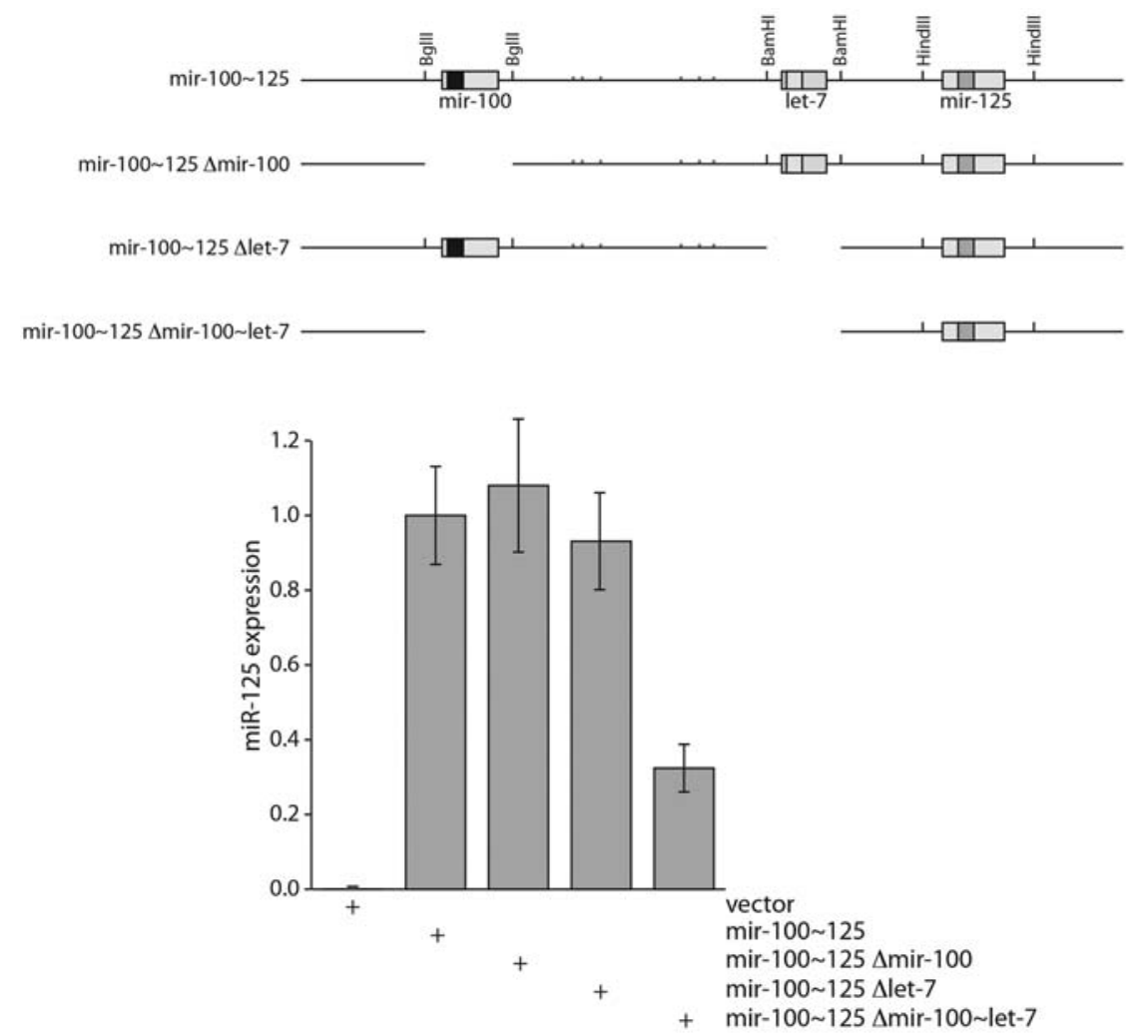

FIGURE 5. Cis-regulation is not limited to the mir-11 998 cluster. S2R+ cells were transfected with constructs expressing wild-type and mutant mir-100 125, as shown in the diagram. RNA extracts were made $2 \mathrm{~d}$ after transfection and mature miR-125, miR-11, miR-998, and bantam were measured by TaqMan assay. miR-125 expression was normalized to miR-11 and miR-998 and to bantam expression. Expression was normalized to wild-type mir-100 125. Error bars represent standard error. Restriction sites were introduced flanking miRNAs for the purpose of generating miRNA deletions. Additional mutations were introduced to facilitate construction of gBlocks, and are indicated by tick marks. See Supplemental Figure S1 for full sequences. stand biological significance of why expression of miR-998 is so tightly linked to expression of miR-11. Strikingly, breaking up the regulation within the mir-11 998 cluster and expressing unchecked miR-998 in the absence of miR-11 resulted in a high frequency of pleiotropic phenotypes in $\sim 20 \%$ of animals. Notably, these phenotypes are not due to the absence of miR-11 because they are suppressed by the loss of miR998. The reason for such pleiotropy is unclear but it is consistent with the emerging view of miRNAs acting to impose subtle changes in transcript and protein expression, thereby reducing the overall noise in gene expression (Baek et al. 2008; Selbach et al. 2008; Mukherji et al. 2011). It is conceivable that unchecked miR-998 would affect the "precision" of the expression of its target genes that would eventually lead to a wide range of phenotypic effects. This explanation is supported by gene expression profiling that revealed a large number of differentially expressed genes of seemingly unrelated GOBP categories to be enriched in the mir-11 998 ${ }^{R M C E}$ mutants. Furthermore, overexpression of miR-998 with several Gal4 drivers resulted in highly penetrant developmental defects indicating that, at least in overexpression settings, miR-998 can affect developmental pathways. Collectively

measured from the ssRNA stem-loop junction (Han et al. 2006). Examination of the predicted structure of longer miRNAs including miR-998 using the M-fold folding algorithm (Zuker 2003) reveals a number of different conformations with similar free energies (data not shown). It is conceivable that the longer length of these miRNAs renders recognition and cleavage by the microprocessor more challenging. Therefore, longer miRNAs may be dependent on more canonical miRNAs in the vicinity for processing. This idea is supported by the finding that miR-998 expression became independent of the presence of an adjacent miRNA when miR-998 was expressed from the shorter mir-1 scaffold. Similarly, the mir-125 miRNA is longer than average, and its expression was significantly compromised when the two other miRNAs in the cluster, mir-100 and let-7, were deleted. This raises a number of questions: How then are long monocistronic miRNAs expressed? What is the consequence of coordinated versus differential expression of miRNAs generated from polycistrons?

We considered addressing the latter question to be particularly important in this work because it would help to under- our data argue the functional importance of tightly linking the expression of miR-998 to the presence of miR-11.

Another important finding that we report here is that the intrinsic regulation within the miRNA clusters does not appear to be limited to the mir-11 998 cluster as the expression of miR-125 from the mir-100 let-7 125 cluster is similarly dependent on mir-100 and let-7. Transcription of a polycistronic pri-miRNA permits coordinated expression of individual mature miRNAs. However, each step in miRNA biogenesis can be modulated, as can the steady-state level of the mature miRNA (Kim et al. 2009). For example, the individual pre-miRNAs from the mir-17 92 cluster are differentially regulated by RNA-binding proteins: hnRNP A1 specifically binds pri-mir- 18 and enhances mir- 18 processing by Drosha (Guil and Cáceres 2007). Therefore, like posttranscriptional regulation of protein expression, miRNA expression can be fine-tuned in response to different biological cues, thereby affecting the timing and extent of miRNA target regulation.

Polycistronic miRNAs can give rise to miRNAs from the same or different miRNA families. These miRNAs can share 
targets and functions or target genes with opposing functions. For example, the oncogenic mir-17 92 cluster encodes miRNAs that target both positive and negative cell cycle regulators and pro- and anti-apoptotic proteins (O'Donnell et al. 2005; Hossain et al. 2006; Zhang et al. 2006; Shan et al. 2009; Li et al. 2012). Moreover, miR-92a and miR-17 have antagonistic effects: Although overexpression of miR92a in mice caused erythroleukemia, coexpression of miR17 abrogated this effect (Li et al. 2012). Similarly to the context-dependent differences in function of miRNAs from the mir-17 92 cluster, we show here that miR-11 and miR-998 carry different functions. The functional consequences of coordinated or differential expression of the different individual miRNAs can determine very different biological outcomes. The impact of this layer of regulation on normal or perturbed developmental processes, as well as potential for therapeutic intervention awaits further investigation.

\section{MATERIALS AND METHODS}

\section{Fly stocks}

All fly crosses were done at $25^{\circ} \mathrm{C}$. The following stocks were obtained from Bloomington Drosophila Stock Center at Indiana University: egfr ${ }^{E 1}$. The following stocks were previously published: mir-11 1 (Truscott et al. 2011); mir-998 ${ }^{\text {exc222 }}$ (Truscott et al. 2014); rbf1 ${ }^{120 a}$ ey-FLP/FM7 Act-GFP; FRT 82B Ubi-GFP (Moon et al. 2006); mir$11^{K O(w-)}$ and mir-11 ${ }^{K O(w+)}$ (Ge et al. 2012); DE-Gal4 (Morrison and Halder 2010).

\section{Construction of cell culture expression vectors}

Insect cell culture expression constructs were generated using the pIEx-7 Ek/LIC kit (Novagen). Ligation-independent cloning-competent inserts were generated as gBlocks (IDT). Insert sequences are in Supplemental Figure S1.

\section{Cell culture, dsRNA knockdown, transfection, and luciferase assay}

$\mathrm{S} 2 \mathrm{R}+$ cells were cultured in Schneider's Insect Medium (Gibco) + $10 \%$ FBS. dsRNA knockdown was done as described in Ambrus et al. (2009). Three days after initial soaking, cells were split and the following day, cells were transfected with the X-tremeGENE HP transfection reagent (Roche) according to manufacturer's protocol. Cells were harvested $24-48 \mathrm{~h}$ post-transfection. Renilla luciferase activity was measured using the Dual Luciferase Assay protocol (Promega).

\section{Recombinase-mediated cassette exchange}

piB-mir-1/998 and piB-mCherry shmiR, mir-998 donor plasmids were generated by replacing the GFP sequence from piB-GFP (Bateman et al. 2006). GFP sequence was removed with BamHI restriction sites and then filled in with T4 DNA polymerase. mir-1/998 and mCherry shmiR, mir-998 were removed from pIEx-7 constructs by restriction digest and filling in with T4 DNA polymerase (see Supplemental Fig. S1 for sequence information). Embryo injec- tions were done at The Best Gene, Inc. Donor plasmid DNA was injected into $\mathrm{F}_{1}$ from cross of $w$; mir-11 $p K O w^{+} / T M 3$ to M\{vas-int. $D m\} Z H-2 A$ (FBti0099694). Progeny were screened for loss of mini-white marker as described in Weng et al. (2009) and then sequenced to confirm insertion.

\section{Northern blot analysis}

Ten micrograms of total RNA extracted from third instar larvae using TRIzol (Invitrogen) was run on a 16\% polyacrylamide gel with 7 $\mathrm{M}$ urea. Resolved RNA was transferred to Zeta-Probe membrane (Bio-Rad), followed by UV crosslinking, and prehybridizing in ULTRAhyb-Oligo (Invitrogen) at $42^{\circ} \mathrm{C}$. RNA oligos (IDT) were $5^{\prime}$-end-labeled with $\left[\gamma^{-}{ }^{32} \mathrm{P}\right]$ ATP using T4 polynucleotide kinase (NEB). Hybridization was performed overnight at $42^{\circ} \mathrm{C}$, followed by two washes in $2 \times$ SSC with $0.1 \%$ SDS, and one wash in $0.5 \times$ SSC with $0.1 \%$ SDS. Membranes were stripped of probes using boiling $0.1 \times$ SSC with $0.1 \%$ SDS.

\section{Immunohistochemistry}

Antibodies used were as follows: rabbit anti-C3 (Cleaved Caspase3), lot 26, 1:100 (Cell Signaling), rat anti-ELAV 1:50, (Developmental Studies Hybridoma Bank), rabbit anti-Pnt P1 (1:200) (Alvarez et al. 2003), rabbit anti-Atonal (1:2000) (from Y. Jan), and Cy3-, and Cy5- conjugated anti-mouse, and anti-rabbit secondary antibodies (Jackson Immunoresearch Laboratories). Immunofluorescence was done as previously described (Ambrus et al. 2009) using a Zeiss Confocal microscope and images were prepared using Adobe Photoshop CS4. All images are confocal single-plane images unless otherwise stated as projection images.

\section{miRNA target prediction}

Comprehensive lists of predicted miR-998 targets and miR-11 targets were compiled from TargetScan (Ruby et al. 2007), MinoTar (Schnall-Levin et al. 2010), PITA (Kertesz et al. 2007), miRanda (Enright et al. 2003), and RNAhybrid (Rehmsmeier et al. 2004), and can be found in Truscott et al. (2014).

\section{Gene expression analysis}

For qRT-PCR, total RNA was isolated from 10 third instar larvae, or 30-50 third instar larval eye discs, with TRIzol (Invitrogen). Reverse transcription to measure standard mRNAs was performed using the SensiFAST cDNA Synthesis Kit (Bioline) according to the manufacturer's specifications. Quantitative PCR was performed with SensiFast SYBR No-Rox Mix (Bioline) info on a Roche Light Cycler 480. Mature miRNA expression was measured by TaqMan hydrolysis probe assay (Applied Biosystems) using the SensiFAST Probe No-ROX Kit (Bioline).

For genome-wide expression analysis, RNA was harvested from 30-50 third instar larval eye discs in biological duplicate from Canton S, mir-11 ${ }^{K O(w-)}$, mir-11 998 ${ }^{R M C E-1}$, and mir-11 998 $8^{R M C E-2}$ and processed for Illumina HiSeq2000 Next Gen RNA Sequencing at the University of Chicago Genomics Facility. Data analysis is described in Supplementary Methods. RNA-seq data files are publicly available through the Gene Expression Omnibus (GEO; Accession number GSE73946). 


\section{SUPPLEMENTAL MATERIAL}

Supplemental material is available for this article.

\section{ACKNOWLEDGMENTS}

We thank Nuria Lopez-Bigas, Barcelona Biomedical Research Park, Spain, for providing the cluster computing facility. We are grateful to Steve Cohen and the Bloomington Stock Center for fly stocks. We thank Zain Paroo for sharing protocols, equipment, and reagents for Northern blot experiments. We thank Jason Bateman for the piB-GFP plasmid, and Jim Skeath for the anti-Pnt P1 antibody. We are thankful to Steve Cohen and Zain Paroo for discussions. This research was supported by grants GM110018 and GM093827 from the National Institutes of Health to M.V.F., by a Scholar Award from the Leukemia \& Lymphoma Society to M.V.F., and by a Postdoctoral Fellowship to M.T. from the Fonds de Recherches en Santé Québec.

Received July 12, 2015; accepted October 14, 2015.

\section{REFERENCES}

Alvarez AD, Shi W, Wilson BA, Skeath JB. 2003. pannier and pointedP2 act sequentially to regulate Drosophila heart development. Development 130: 3015-3026.

Ambrus AM, Rasheva VI, Nicolay BN, Frolov MV. 2009. Mosaic genetic screen for suppressors of the de2f1 mutant phenotype in Drosophila. Genetics 183: 79-92.

Ameres SL, Zamore PD. 2013. Diversifying microRNA sequence and function. Nat Rev Mol Cell Biol 14: 475-488.

Baek D, Villén J, Shin C, Camargo FD, Gygi SP, Bartel DP. 2008. The impact of microRNAs on protein output. Nature 455: 64-71.

Bateman JR, Lee AM, Wu CT. 2006. Site-specific transformation of Drosophila via $\phi \mathrm{C} 31$ integrase-mediated cassette exchange. Genetics 173: 769-777.

Ebert MS, Sharp PA. 2012. Roles for microRNAs in conferring robustness to biological processes. Cell 149: 515-524.

Enright AJ, John B, Gaul U, Tuschl T, Sander C, Marks DS. 2003. MicroRNA targets in Drosophila. Genome Biol 5: R1.

Ge W, Chen Y-W, Weng R, Lim SF, Buescher M, Zhang R, Cohen SM. 2012. Overlapping functions of microRNAs in control of apoptosis during Drosophila embryogenesis. Cell Death Differ 19: 839-846.

Guil S, Cáceres JF. 2007. The multifunctional RNA-binding protein hnRNP A1 is required for processing of miR-18a. Nat Struct Mol Biol 14: 591-596.

Haley B, Hendrix D, Trang V, Levine M. 2008. A simplified miRNAbased gene silencing method for Drosophila melanogaster. Dev Biol 321: 482-490.

Han J, Lee Y, Yeom K-H, Nam J-W, Heo I, Rhee J-K, Sohn SY, Cho Y, Zhang B-T, Kim VN. 2006. Molecular basis for the recognition of primary microRNAs by the Drosha-DGCR8 complex. Cell 125: 887-901.

Hornstein E, Shomron N. 2006. Canalization of development by microRNAs. Nat Genet 38: S20-S24.

Hossain A, Kuo MT, Saunders GF. 2006. Mir-17-5p regulates breast cancer cell proliferation by inhibiting translation of AIB1 mRNA. Mol Cell Biol 26: 8191-8201.

Kertesz M, Iovino N, Unnerstall U, Gaul U, Segal E. 2007. The role of site accessibility in microRNA target recognition. Nat Genet 39: $1278-1284$.

Kim VN, Han J, Siomi MC. 2009. Biogenesis of small RNAs in animals. Nat Rev Mol Cell Biol 10: 126-139.
Kozomara A, Griffiths-Jones S. 2011. miRBase: integrating microRNA annotation and deep-sequencing data. Nucleic Acids Res 39: D152-D157.

Lee Y, Jeon K, Lee J-T, Kim S, Kim VN. 2002. MicroRNA maturation: stepwise processing and subcellular localization. EMBO $J$ 21: 4663-4670.

Lee Y, Ahn C, Han J, Choi H, Kim J, Yim J, Lee J, Provost P, Rådmark O, Kim S, et al. 2003. The nuclear RNase III Drosha initiates microRNA processing. Nature 425: 415-419.

Li Y, Vecchiarelli-Federico LM, Li Y-J, Egan SE, Spaner D, Hough MR, Ben-David Y. 2012. The miR-17-92 cluster expands multipotent hematopoietic progenitors whereas imbalanced expression of its individual oncogenic miRNAs promotes leukemia in mice. Blood 119: $4486-4498$.

Moon N-S, Di Stefano L, Dyson N. 2006. A gradient of epidermal growth factor receptor signaling determines the sensitivity of rbf1 mutant cells to E2F-dependent apoptosis. Mol Cell Biol 26: 7601-7615.

Morrison CM, Halder G. 2010. Characterization of a dorsal-eye Gal4 Line in Drosophila. Genesis 48: 3-7.

Mukherji S, Ebert MS, Zheng GXY, Tsang JS, Sharp PA, van Oudenaarden A. 2011. MicroRNAs can generate thresholds in target gene expression. Nat Genet 43: 854-859.

O'Donnell KA, Wentzel EA, Zeller KI, Dang CV, Mendell JT. 2005. cMyc-regulated microRNAs modulate E2F1 expression. Nature 435: 839-843.

Rehmsmeier M, Steffen P, Höchsmann M, Giegerich R. 2004. Fast and effective prediction of microRNA/target duplexes. RNA 10: 1507-1517.

Ruby JG, Stark A, Johnston WK, Kellis M, Bartel DP, Lai EC. 2007. Evolution, biogenesis, expression, and target predictions of a substantially expanded set of Drosophila microRNAs. Genome Res 17: 1850-1864.

Schmiedel JM, Klemm SL, Zheng Y, Sahay A, Blüthgen N, Marks DS, van Oudenaarden A. 2015. Gene expression. MicroRNA control of protein expression noise. Science 348: 128-132.

Schnall-Levin M, Zhao Y, Perrimon N, Berger B. 2010. Conserved microRNA targeting in Drosophila is as widespread in coding regions as in 3' UTRs. Proc Natl Acad Sci 107: 15751-15756.

Selbach M, Schwanhäusser B, Thierfelder N, Fang Z, Khanin R, Rajewsky N. 2008. Widespread changes in protein synthesis induced by microRNAs. Nature 455: 58-63.

Shan SW, Lee DY, Deng Z, Shatseva T, Jeyapalan Z, Du WW, Zhang Y, Xuan JW, Yee S-P, Siragam V, et al. 2009. MicroRNA MiR-17 retards tissue growth and represses fibronectin expression. Nat Cell Biol 11: 1031-1038.

Sokol NS, Xu P, Jan Y-N, Ambros V. 2008. Drosophila let-7 microRNA is required for remodeling of the neuromusculature during metamorphosis. Genes Dev 22: 1591-1596.

Truscott M, Islam ABMMK, López-Bigas N, Frolov MV. 2011. mir-11 limits the proapoptotic function of its host gene, dE2f1. Genes Dev 25: $1820-1834$.

Truscott M, Islam ABMMK, Lightfoot J, López-Bigas N, Frolov MV. 2014. An intronic microRNA links Rb/E2F and EGFR signaling ed. G. Bosco. PLoS Genet 10: e1004493.

Weng R, Chen Y-W, Bushati N, Cliffe A, Cohen SM. 2009. Recombinase-mediated cassette exchange provides a versatile platform for gene targeting: knockout of miR-31b. Genetics 183: 399-402.

Zhang L, Huang J, Yang N, Greshock J, Megraw MS, Giannakakis A, Liang S, Naylor TL, Barchetti A, Ward MR, et al. 2006. microRNAs exhibit high frequency genomic alterations in human cancer. Proc Natl Acad Sci 103: 9136-9141.

Zuker M. 2003. Mfold web server for nucleic acid folding and hybridization prediction. Nucleic Acids Res 31: 3406-3415. 

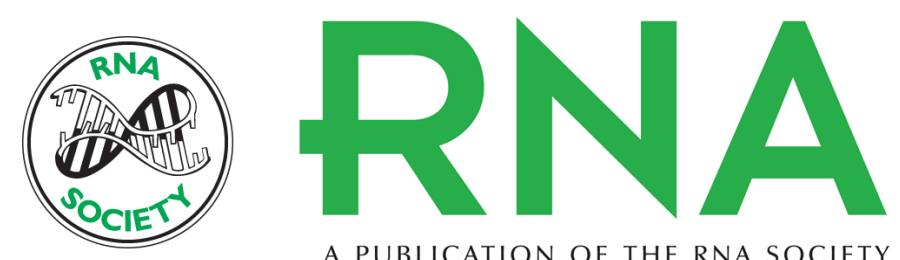

A PUBLICATION OF THE RNA SOCIETY

\title{
Novel regulation and functional interaction of polycistronic miRNAs
}

\author{
Mary Truscott, Abul B.M.M.K. Islam and Maxim V. Frolov
}

RNA 2016 22: 129-138 originally published online November 9, 2015

Access the most recent version at doi:10.1261/rna.053264.115

Supplemental Material

References

Creative Commons License

Email Alerting Service
http://rnajournal.cshlp.org/content/suppl/2015/11/04/rna.053264.115.DC1

This article cites 35 articles, 15 of which can be accessed free at: http://rnajournal.cshlp.org/content/22/1/129.full.html\#ref-list-1

This article is distributed exclusively by the RNA Society for the first 12 months after the full-issue publication date (see http://rnajournal.cshlp.org/site/misc/terms.xhtml). After 12 months, it is available under a Creative Commons License (Attribution-NonCommercial 4.0 International), as described at http://creativecommons.org/licenses/by-nc/4.0/.

Receive free email alerts when new articles cite this article - sign up in the box at the top right corner of the article or click here.

To subscribe to $R N A$ go to:

http://rnajournal.cshlp.org/subscriptions

(C) 2015 Truscott et al.; Published by Cold Spring Harbor Laboratory Press for the RNA Society 